Original Research Paper

\title{
Identification of Human Emotions via Univariate and Multivarite Multiscale Entropy
}

\author{
Kawser Ahammed \\ Department of Electrical and Electronic Engineering (Former Applied Physics and ECE), \\ University of Dhaka, Dhaka, Bangladesh
}

Article history

Received: 05-06-2015

Revised: $17-06-2015$

Accepted: 25-06-2015

Email: kawser.apece@gmail.com

\begin{abstract}
This work analyzes the emotions of human in terms of complexity. This analysis is achieved by applying both univariate and multivariate multiscale entropy methods on a multimodal dataset. Most of the contemporary human-computer interaction systems are unable to identify human affective states. So, the benefit of analyzing human emotions is to fill this gap by detecting human affective states. The univariate and multivariate multiscale entropy analysis curves obtained using multimodal dataset show differences in terms of complexity among different affective states, which can be used for emotion detection and classification for machine vision applications.
\end{abstract}

Keywords: Multimodal Dataset, Affective States Computing, Emotion Classification, Machine Vision Applications

\section{Introduction}

Over the past two decades, research on emotion has increased significantly with many fields including psychology, neuroscience, endocrinology, medicine, history, sociology and even computer science. The different theories that attempted to analyze human emotions explained only the origin, neurobiology, experience and function of emotions. Current areas of research on emotion include the development of materials that stimulate and elicit emotion but do not identify emotion in terms of complexity. For that reason, this work has been performed for the first time in the history of emotion analysis. Emotion is defined as a psycho-physiological process triggered by conscious and/or unconscious perception of an object or situation. In other words, an emotion is usually created by bodily changes, expressions and actions. Emotion is felt as a distinctive type of mental state and caused by a person consciously or unconsciously evaluating an event as relevant to a concern that is important; Emotion can be positive or negative (Oatley et al., 2006) Positive emotion refers to a concern that is advanced. On the other hand, negative emotion is realized as a concern that is impeded. Emotion is expressed through different types of manner such as mood, temperament and motivation. Emotions contribute a significant role in human communication. One can express his emotion either verbally through emotional vocabulary, or by expressing non-verbal cues such as intonation of voice, facial expressions and gestures.

In this article, four types of emotion have been analyzed using both univariate and multivariate multiscale entropy methods. The four types of emotions are valence, dominance, arousal and liking. The range of arousal can be from inactive to active, whereas the range of valence can be from unpleasant to pleasant.

Dominance ranges from a helpless and weak feeling to an empowered feeling. To evaluate the mental condition of a person, emotion assessment is required. This can be performed through analysis of person's emotional expressions and/or physiological signals. Any observable verbal and non-verbal behavior that communicates emotion refers to emotional expressions. So far, most of the studies on emotion assessment have focused on the analysis of facial expressions and speech to determine a person's emotional state. Emotional information that can be used for emotion assessment exists in physiological signals (Koelstra et al., 2012). The univariate multiscale entropy (Costa et al., 2005) measures the complexity of a finite length time series. The univariate multiscale entropy analysis can only consider every data channel separately. The applications of this method include the analysis of pathologic heartbeat conditions like erratic cardiac arrhythmia and congestive heart failure (Costa et al., 2002), Electroencephalogram (EEG) changes in patients with 
Alzheimer's disease (Hornero et al., 2009), postural sway dynamics analysis (Costa et al., 2007) and complex dynamics of human redblood cells (Costa et al., 2008). This method never analyzed human emotions. Multivariate multiscale entropy (Ahmed and Mandic, 2011a) measures the complexity of multichannel data. The possible applications of this method are analysis of brain consciousness (Ahmed et al., 2011), complexity analysis of multichannel data (Ahmed and Mandic, 2011a; 2011b), dynamical complexity of human responses (Ahmed et al., 2012) and human centered complexity Analysis (Looney et al., 2012). This method also never analyzed human emotions.

In this study a multimodal database (Koelstra et al., 2012) containing emotional data has been used to analyze human emotions. To make that database, 40 music video clips were used as the visual stimuli to elicit different emotions. Using these emotional data, univariate sample entropy is calculated in terms of scale factor in order to determine higher complexity emotional state. Similarly, multivariate sample entropy is calculated in terms of scale factor.

\section{Univariate Multiscale Entropy (UMSE) Method}

The UMSE method includes two procedures:

For a given time series, multiple coarse-grained time series are constructed by averaging the data points within non-overlapping windows of increasing length, $\varepsilon$ (scale factor). Suppose one-dimensional discrete time series of length $N=\left\{u_{1}, u_{2}, \ldots, u_{i}, \ldots, u_{N}\right\}$ with a constant time interval, $\tau$, is given. From this time series consecutive coarse-grained time series, $\left\{x^{(\varepsilon)}\right\}$ is constructed, corresponding to the scale factor, $\varepsilon$. Usually, each element of a coarse-grained time series is obtained by the following Equation 1:

$$
x_{j}^{(\varepsilon)}=\frac{1}{\varepsilon} \sum_{i=(j-1) \varepsilon+1}^{j \varepsilon} u_{i}, 1 \leq j \leq \frac{N}{\varepsilon}
$$

where, $N$ denotes the number of data points or length of time series and $\varepsilon$ represents the scale factor.

Sample entropy (SampEn) is calculated for each coarse-grained time series and then plotted as a function of the scale factor $\varepsilon$. For Multivariate Multiscale Entropy (MMSE) method, $u_{i}=u_{l, i}$ and $l=1,2, \ldots, c$; where $c$ denotes the number of channels.

\section{Selection of Parameter Values}

During calculation of sample entropy for univariate and multivariate, several parameters have to be chosen introducing their own constraints. For example, for multivariate data, the individual channels exhibit different embedding parameters $m_{k}$ and $\tau_{k}$. During sample entorpy and multivariate sample entorpy calculation, the threshold parameter $r$ is set to some percentage of the standard deviation. For univariate and multivariate sample entorpy, $r$ has been taken as 7\% for better performance.

\section{Emotion Analysis Via Univariate Multiscale Entropy}

In this section, zygomaticus electromyogram (zEMG), Trapezius electromyogram (tEMG) and Plethysmograph univariate contents are analyzed in terms of complexity in order to test affective states (i.e., valence, arousal, dominance and liking) of human. For complexity analysis, the highest values of each emotion label are used among the four emotion labels. The highest values of each emotion are given in table 1 . The values of the parameters used to calculate univariate sample entropy were $m_{1}=3, m_{2}=$ 2, $\tau_{1}=1, \tau_{2}=2$.

From Fig. 1a, it is clear that the values of sample entropy for dominance emotion do not overlap with the values of sample entropy for valence emotion for all scale factors. This indicates that both emotions can be identified by UMSE algorithm. Since the values of sample entropy for the dominance emotion are higher than that of valence, dominance is more complex than valence. This fact also implies that human has an empowered feeling (in control of everything) for dominance and unpleasant feeling (e.g., sad, stressed) for valence.

In Fig. 1b, it has been shown that both liking and valence emotions can be identified by UMSE algorithm due to non-overlapping behavior of both emotions for the majority of the scale factors. In this case, Liking emotion is more complex than the valence because of higher entropy values for the majority of the scale factors. This fact also implies that human has a strong liking feeling for liking emotion and unpleasant feeling (e.g., sad, stressed) for valence.

In Fig. 2a, dominance and arousal emotions are clearly distinguishable. This indicates that both emotions can be identified by UMSE algorithm. Due to the higher sample entropy values of dominance than arousal for all scale factors, it can be said that dominance is more complex than arousal. This fact also implies that human has an empowered feeling (in control of everything) for dominance and an inactive feeling (e.g., uninterested, bored) for arousal. Similarly in Fig. 2b, it has been shown that dominance and valence emotions can be identified by UMSE algorithm. 
Table 1. Highest values for four emotion labels

\begin{tabular}{|c|c|c|c|c|}
\hline \multirow[b]{2}{*}{ Video/trial } & \multicolumn{4}{|l|}{ Labels } \\
\hline & Valence & Arousal & Dominance & Liking \\
\hline 1 & 9.0 & & & \\
\hline 2 & & & & 8.08 \\
\hline 10 & & & 9.00 & \\
\hline 12 & & & & 9.00 \\
\hline 16 & & & 8.05 & \\
\hline 17 & 9.0 & & & \\
\hline 19 & 9.0 & & & \\
\hline 23 & & & & 7.82 \\
\hline 28 & & & 7.14 & \\
\hline 29 & & & & 9.00 \\
\hline 31 & & & & 9.00 \\
\hline 32 & & 9.00 & & \\
\hline 33 & & 8.03 & & \\
\hline 37 & & 8.06 & & \\
\hline 38 & & 9.00 & & \\
\hline
\end{tabular}

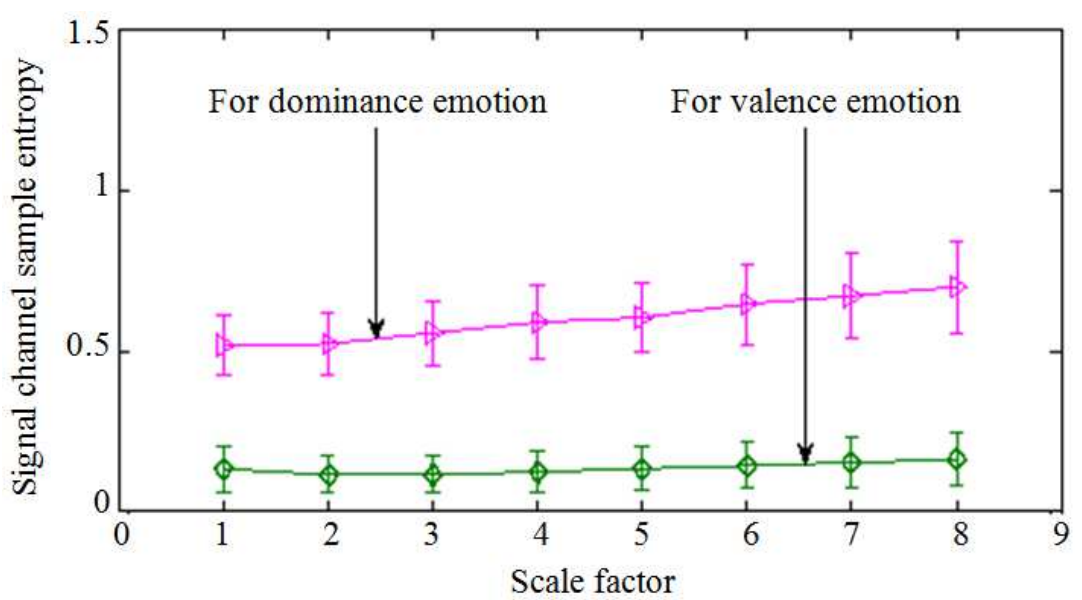

(a)

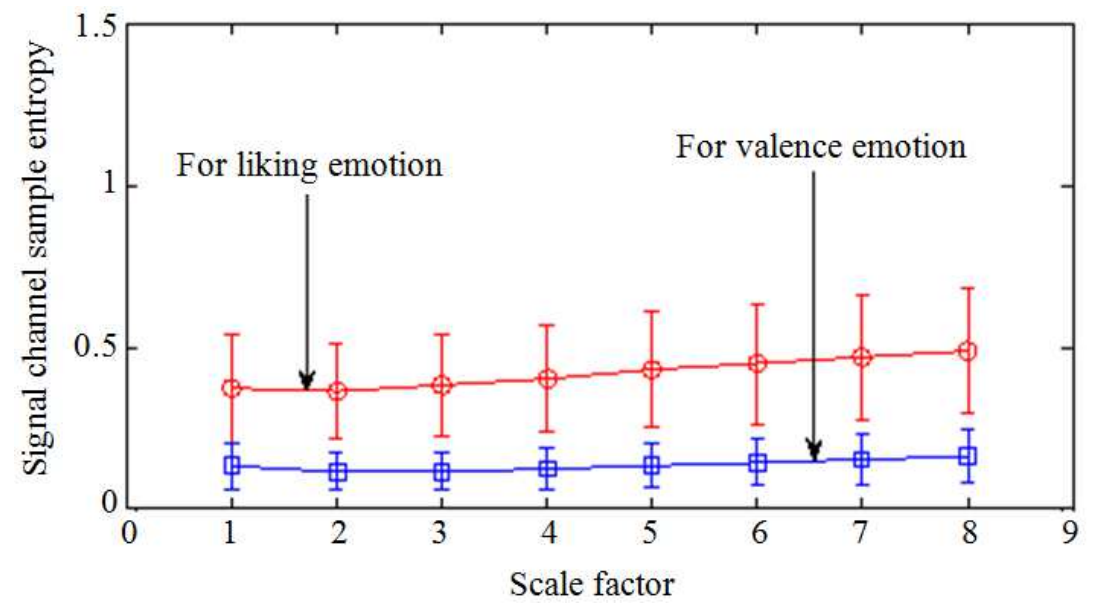

(b)

Fig. 1. Univariate Multiscale Entropy (UMSE) analysis of Zygomaticus Major EMG, zEMG1-zEMG2 (zEMG) time series (mean zero, variance one), each with 7681 data points. Symbols represent an average of 3 independent trials for dominance, 3 independent trials for valence and 5 independent trials for liking. Error bars represent the Standard Deviation (SD). Lines represent numerical evaluation of analytic Univariate Sample Entropy Calculation (a) For dominance and valence emotion (b) For liking and valence emotion 


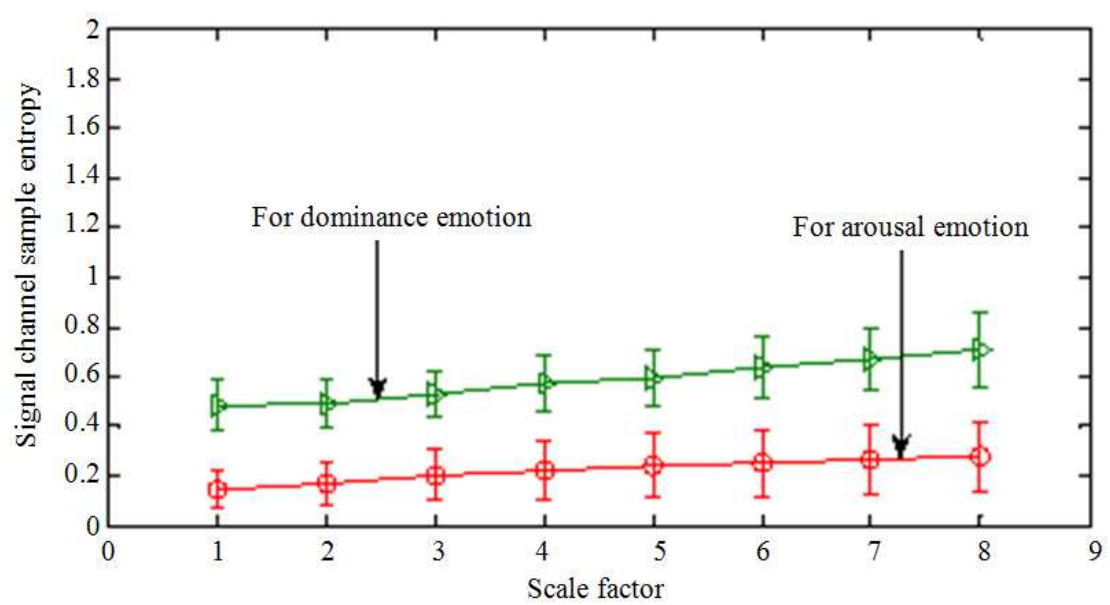

(a)

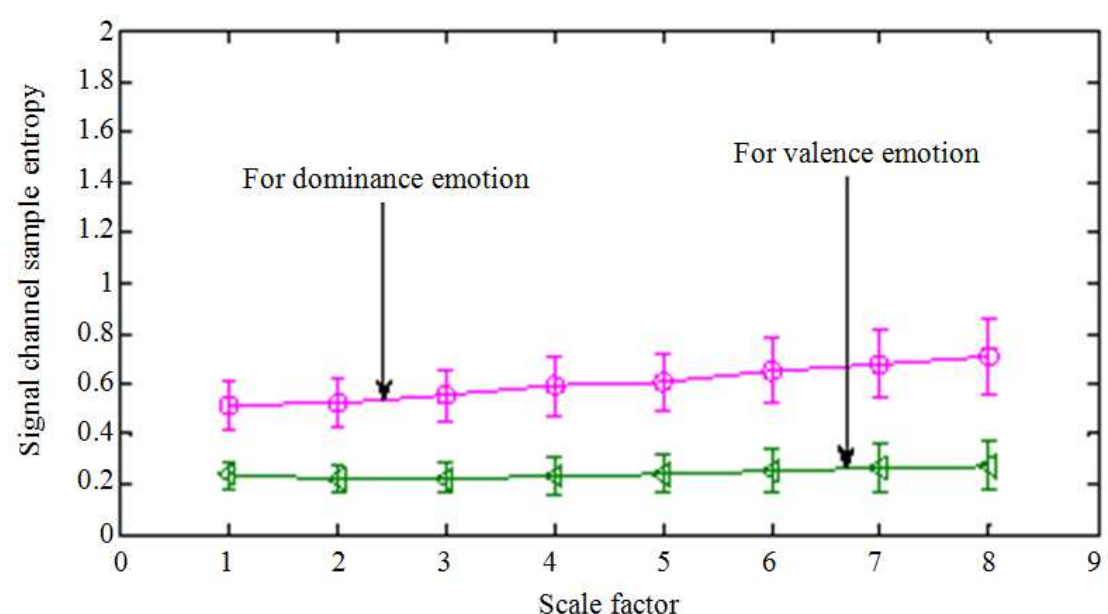

(b)

Fig. 2. Univariate Multiscale Entropy (UMSE) analysis of Trapezius EMG, tEMG1-tEMG2 (tEMG) time series (mean zero, variance one), each with 7681 data points. Symbols represent an average of 3 independent trials for dominance, 4 independent trials for arousal and 3 independent trials for valence. Error bars represent the Standard Deviation (SD). Lines represent numerical evaluation of analytic Univariate Sample Entropy Calculation (a) For dominance and arousal emotion (b) For dominance and valence emotion

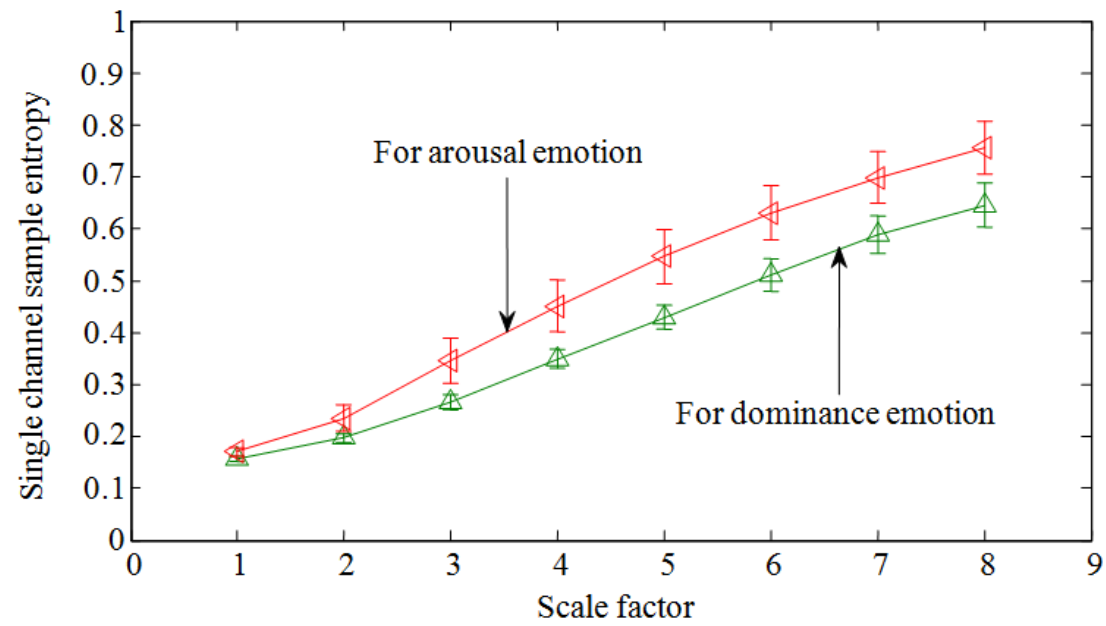

(a) 


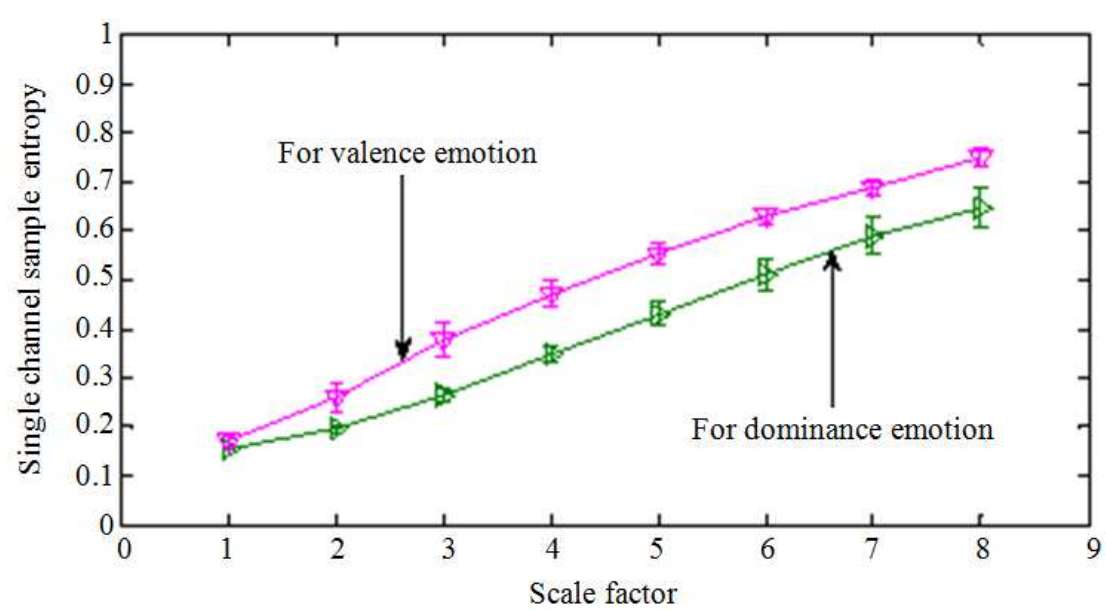

(b)

Fig. 3. Univariate Multiscale Entropy (UMSE) analysis of Plethysmograph time series (mean zero, variance one), each with 7681 data points. Symbols represent an average of 4 independent trials for arousal, 3 independent trials for dominance and 3 independent trials for valence. Error bars represent the standard deviation (SD). Lines represent numerical evaluation of analytic Univariate Sample Entropy Calculation (a) For arousal and dominance emotion (b) For valence and dominance emotion

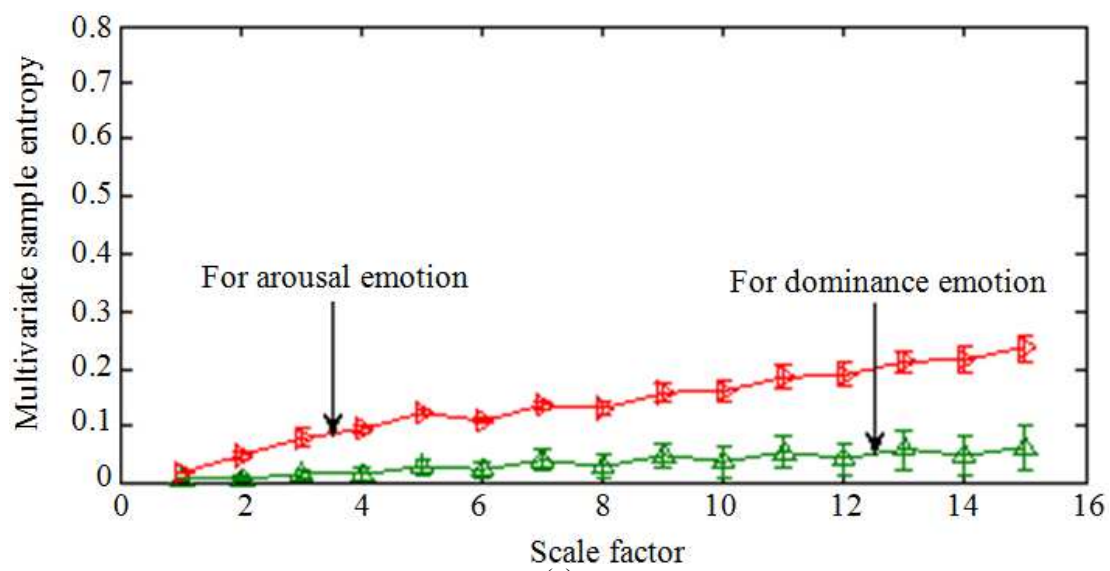

(a)

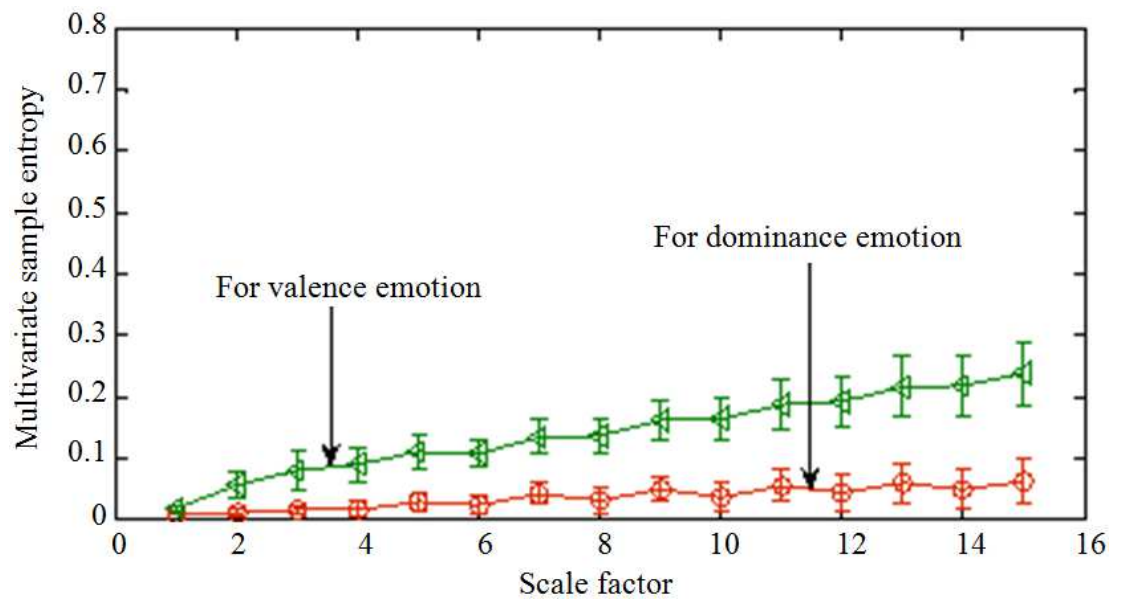

(b)

Fig. 4. Multivariate Multiscale Entropy (MMSE) analysis of Plethysmograph and Temperature time series (mean zero, variance one), each with 7681 data points. Symbols represent an average of 4 independent trials for arousal, 3 independent trials for valence and 3 independent trials for dominance. Error bars represent the Standard Deviation (SD). Lines represent numerical evaluation of analytic multivariate sample entropy calculation (a) For arousal and dominance emotion (b) For valence and dominance emotion 
From Fig. 3a, one can states that the values of sample entropy of arousal emotion are higher than that of dominance except for scale factor 1 and 2. Both emotions do not overlap with each other except scale factor 1 and 2. Due to non-overlapping behavior for the majority of the scale factors, both emotions can be identified by UMSE algorithm. In addition to, arousal emotion is more complex than dominance. This fact also implies that human an active feeling (e.g., alert, excited) for arousal and a helpless and weak feeling (without control) for dominance.

In Fig. 3b, it is noticeable that the valence and dominance emotions are distinguishable except scale factor 1. Both emotions do not overlap with each other except scale factor 1 . This indicates that both emotions can be identified by UMSE algorithm. In addition to, valence emotion is more complex than dominance. This fact also implies that human has a pleasant (e.g., happy, elated) feeling for valence and a helpless, weak feeling (without control) for dominance.

\section{Emotion Analysis Via Multivariate Multiscale Entropy}

This section analyzes the PlethysmographTemperature multivariate contents in terms of complexity in order to test affective states of human. The values of the parameters used to calculate multivariate sample entropy were $m_{1}=3, m_{2}=2, \tau_{1}=1, \tau_{2}=2$.

From Fig. 4a, one can says that the values of sample entropy for the dominance and arousal emotion increase with the increase of scale factors except for scale factor 1. Both of emotions do not overlap with each other except scale factor 1 . This indicates that both emotions can be identified by MMSE algorithm. Since the values of sample entropy for the arousal emotion are higher than the dominance except for scale factor 1 , it can be said that, unlike dominance, arousal emotion contains correlations across multiple time scales and is, therefore, more complex than dominance. This fact also implies that human has an active (e.g., alert, excited) feeling for arousal and helpless, weak (without control) feeling for dominance.

In Fig. $4 b$, for scale factor $\geq 2$, the values of sample entropy for the dominance and valence emotions increase. Both of them do not overlap with each other except scale factor 1 . This indicates that both emotions can be identified by MMSE algorithm. Since the values of sample entropy for the valence emotion are higher than the dominance, it can be said that, unlike dominance, valence emotion contains correlations across multiple time scales and is, therefore, more complex than dominance. This fact also implies that human has a pleasant feeling (e.g., happy, elated) for valence and helpless, weak (without control) feeling for dominance.

\section{Discussion}

The analysis of human emotion analyzed above via univariate and multivariate multiscale entropy obviously shows that affective states of human emotion can be identified by both univariate and multivariate multiscale entropy algorithms because of nonoverlapping characteristic of emotions. This analysis will help in detecting human emotion and classification for future machine vision applications. Therefore, univariate and multivariate multiscale entropy methods can be used to analyze human emotions for the first time in the history of emotion analysis.

\section{Conclusion}

This work applies both univariate and multivariate multiscale entropy method on a multimodal dataset for the analysis of spontaneous emotions using physiological signals. The goal of this work is to resolve the lack of emotional intelligence suffered from the humancomputer interaction systems in a practical scenario. In this study, both univariate and multivariate sample entropies are calculated for different emotions such as valence, arousal, dominance and liking using physiological signals and then are compared to measure which emotion has the higher complexity than the other in order to detect human affective states. So, these analysis results of human emotion can be implemented as a reference in a practical scenario to identify human affective states for the first time.

\section{Acknowledgment}

The author would like to graciously thank Dr. Mosabber Uddin Ahmed, Associate Professor, Dept. of EEE, University of Dhaka, Bangladesh, because of supporting to this research.

\section{Ethics}

This work is original and has been done for the first time.

\section{References}

Ahmed, M.U. and D.P. Mandic, 2011a. Multivariate multiscale entropy analysis. IEEE Signal Process. Lett., 19: 91-94. DOI: 10.1109/LSP.2011.2180713

Ahmed, M.U. and D.P. Mandic, 2011b. Multivariate multiscale entropy: A tool for complexity analysis of multichannel data. Phys. Rev. E, 84:1-10. DOI: 10.1103/PhysRevE.84.061918 
Ahmed, M.U., L. Li, J. Cao and D.P. Mandic, 2011. Multivariate multiscale entropy for brain consciousness analysis. Proceeding of the Annual International Conference of the IEEE Engineering in Medicine and Biology Society, Aug. 30-Sept. 3, IEEE Xplore Press, Boston, pp: 810-813. DOI: 10.1109/IEMBS.2011.6090185

Ahmed, M.U., N. Rehman, D. Looney, T. Rutkowski and D.P. Mandic, 2012. Dynamical complexity of human responses: A multivariate data-adaptive framework. Bull. Polish Acad. Sci., 60: 433-445. DOI: $10.2478 / \mathrm{v} 10175-012-0055-0$

Costa, M., A.A. Priplata, L.A. Lipsitz, Z. Wu and N.E. Huang et al., 2007. Noise and poise: Enhancement of postural complexity in the elderly with a stochastic-resonance-based therapy. Europhys. Lett., 77: 1-5. DOI: 10.1209/0295-5075/77/68008

Costa, M., A.L. Goldberger and C.K. Peng, 2002. Multiscale entropy analysis of complex physiologic time series. Phys. Rev. Lett., 89: 1-4. DOI: 10.1103/PhysRevLett.89.068102

Costa, M., A.L. Goldberger and C.K. Peng, 2005. Multiscale entropy analysis of biological signals. Phys. Rev. E, 71: 1-18.

DOI: 10.1103/PhysRevE.71.021906
Costa, M., I. Ghiran, C.K. Peng, A. Nicholson-Weller and A.L. Goldberger, 2008. Complex dynamics of human red blood cell flickering: Alterations with in vivo aging. Phys. Rev. E, 78: 020901-020901. DOI: 10.1103/PhysRevE.78.020901

Hornero, R., D. Absolo, J. Escudero and C. Gomez, 2009. Nonlinear analysis of electroencephalogram and magnetoencephalogram recordings in patients with Alzheimer's disease. Philos. Trans. A Math. Phys. Eng. Sci., 367: 317-36.

DOI: $10.1098 /$ rsta.2008.0197.

Koelstra, S., C. Muehl, M. Soleymani, J.S. Lee and A. Yazdani et al., 2011. DEAP: A database for emotion analysis; using physiological signals. IEEE Trans Affective Comput., 3: 18-31. DOI: 10.1109/T-AFFC.2011.15

Looney, D., M.U. Ahmed and D.P. Mandic, 2012. Human-centred multivariate complexity analysis. Natural Intell.: INNS Magazine, 1: 40-43.

Oatley, K., D. Keltner and J.M. Jenkins, 2006. Understanding Emotions. 2nd Edn., Wiley, Malden, ISBN-10:1405131039, pp: 510. 